\title{
Processing of Dielectric Optical Coatings by Nanosecond and Femtosecond UV Laser Ablation
}

\author{
J. Ihlemann, J. Békési, J.-H. Klein-Wiele, and P. Simon \\ Laser-Laboratorium Göttingen e.V., Hans-Adolf-Krebs-Weg 1, 37077 Göttingen, Germany \\ Correspondence should be addressed to J. Ihlemann, juergen.ihlemann@llg-ev.de \\ Received 31 May 2008; Accepted 2 September 2008 \\ Recommended by Jacques Albert
}

\begin{abstract}
Microprocessing of dielectric optical coatings by UV laser ablation is demonstrated. Excimer laser ablation at deep UV wavelengths $(248 \mathrm{~nm}, 193 \mathrm{~nm})$ is used for the patterning of thin oxide films or layer stacks. The layer removal over extended areas as well as sub- $\mu$ m-structuring is possible. The ablation of $\mathrm{SiO}_{2}, \mathrm{Al}_{2} \mathrm{O}_{3}, \mathrm{HfO}_{2}$, and $\mathrm{Ta}_{2} \mathrm{O}_{5}$ layers and layer systems has been investigated. Due to their optical, chemical, and thermal stability, these inorganic film materials are well suited for optical applications, even if UV-transparency is required. Transparent patterned films of $\mathrm{SiO}_{2}$ are produced by patterning a UV-absorbing precursor $\mathrm{SiO}_{x}$ suboxide layer and oxidizing it afterwards to $\mathrm{SiO}_{2}$. In contrast to laser ablation of bulk material, in the case of thin films, the layer-layer or layer-substrate boundaries act as predetermined end points, so that precise depth control and a very smooth surface can be achieved. For large area ablation, nanosecond lasers are well suited; for patterning with submicron resolution, femtosecond excimer lasers are applied. Thus the fabrication of optical elements like dielectric masks, pixelated diffractive elements, and gratings can be accomplished.
\end{abstract}

Copyright (C) 2008 J. Ihlemann et al. This is an open access article distributed under the Creative Commons Attribution License, which permits unrestricted use, distribution, and reproduction in any medium, provided the original work is properly cited.

\section{Introduction}

Optical coatings have a variety of applications. Especially design, fabrication, and applications of dielectric optical interference coatings are subject of numerous studies [1]. For the classical use as mirror, beam splitter, or antireflection coating, extended areas have to be homogeneously coated. Therefore, the production of dielectric coatings has been optimized to obtain such high quality coatings nearly without defects on large substrates. But there are other applications, where the coating is needed in locally well-defined areas, for example, masks or waveguides. Coating technology is not well developed in this direction. Deposition through stencil masks is possible but not with high spatial resolution. In this case, the coatings have to be processed following the deposition process in order to generate spatially well-defined patterns. Usually lithographic processes are applied, but they have limited applicability, because the required etching processes are complicated and not sufficiently developed for all used materials.

Laser ablation is a versatile and widespread method for microprocessing of materials. Nearly any kind of technical materials like polymers, metals, glass, ceramics, and composite materials has been investigated with respect to its response to intense pulsed laser irradiation $[2,3]$. In the field of high-precision microfabrication, especially (UV-) excimer lasers are used due to their inherent capability of producing high spatial resolution. Various applications like drilling of micro holes or nozzles, marking of eye glasses, or stripping of wires have been developed and transferred into industrial processes.

But laser ablation is not only good for the treatment of bulk material, it can be utilized for the patterning of thin films as well, for example, optical coatings. In contrast to the treatment of bulk materials, where many laser pulses are required to, for example, drill a material to a certain depth, in the case of thin layers very few or even a single pulse is sufficient to generate the desired ablation pattern. Regarding the capability of simultaneous processing of large areas by mask projection, which is enabled by the flat top beam profile of excimer lasers, there seems to be a huge potential of patterning thin films by this method.

Because under certain conditions the layer-layer or layersubstrate boundaries act as predetermined breaking points, 
the ablated depth profile is not directly correlated with the (sometimes inhomogeneous) beam profile. Whereas in the case of bulk material ablation, spatial variations of the beam intensity will lead to a correspondingly irregular surface profile, this will not necessarily be the case for layer ablation, because there is a certain process window concerning the fluence for complete layer ablation [4]. Thus even with some spatial variations of the irradiation fluence, a very flat surface can be achieved.

Patterning of optical layers is useful for the fabrication of optical components like waveguides, coupling gratings, refractive, reflective, or diffractive structures. In most cases, transparent films with high transmission in the operation wavelength range have to be used. For applications in the visible or infrared spectral ranges, often polymeric materials can be applied, though their mechanical and thermal stability is limited. For example, the laser patterning of layers for fabricating (diffractive) optical elements has already been performed with polyimide films [5]. For UV applications, the control of processing UV-transparent materials, especially inorganic oxide materials, is necessary. Within the broad range of methods for the microfabrication of optical elements, this paper treats the structuring of (UV-) transparent inorganic oxide layers and layer stacks by laser ablation. Special attention is paid to a combined process, where an absorbing suboxide ( $\mathrm{SiO}$ ) layer is patterned and afterwards oxidized into a transparent oxide $\left(\mathrm{SiO}_{2}\right)$.

\section{Methods and Materials}

\subsection{Laser Systems}

Excimer lasers are optimally suited for a number of tasks concerning ablation of optical coatings. The emitted UVlight is readily absorbed by most of the relevant coating materials. However, due to the specific band edge of each material, different laser wavelengths are required. $\mathrm{TiO}_{2}$, for example, s already absorbed in the near UV $(308 \mathrm{~nm})$. Efficient absorption of $\mathrm{Ta}_{2} \mathrm{O}_{5}$ is obtained at $248 \mathrm{~nm}$, and for $\mathrm{HfO}_{2}$ an even shorter wavelength of $193 \mathrm{~nm}$ is required. Excimer lasers emit powerful pulses with a flat top beam profile enabling large area processing, for example, by mask projection. Even the pulse duration of 10 or 20 nanosecond seems to be quite optimal for the ablation of optical layers. The thermal diffusion length $\mathrm{L}$ of around 50 to $500 \mathrm{~nm}$ which is related to the laser pulse length $\tau$ by $\mathrm{L} \sim \tau^{1 / 2}$ and characterizes the heat-affected zone (HAZ) is short enough to minimize lateral damage, but sufficiently long to provide heat flow within the typical layer thickness, which promotes the liftoff of a complete layer with a single pulse $[6,7]$. However, when structure details in the submicron range have to be fabricated, for example, gratings with a period of several $100 \mathrm{~nm}$, this thermal diffusion length is too large, so that shorter laser pulses are required. To combine the capability of high optical resolution with that of a small heat-affected zone, a UV ultrafast laser system is applied. This comprises a Ti:Sa front-end laser, a frequency tripling unit to convert the wavelength of the ultrashort pulses into the UV spectral range, and a specially designed $\mathrm{KrF}$ amplifier to boost up

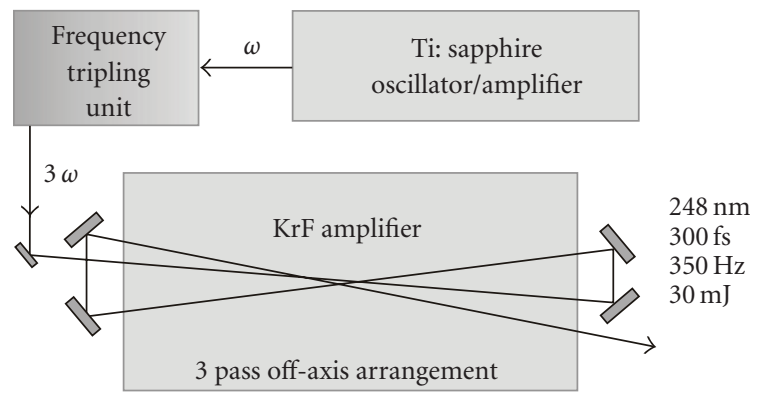

FIgURE 1: Layout of the UV femtosecond hybrid laser system.

the energy of the pulses to the several $\mathrm{mJ}$ range. The current laser arrangement (Figure 1) uses a commercial Ti:Sa frontend system delivering pulses of 150 femtoseconds duration at a wavelength of $745 \mathrm{~nm}$. After frequency tripling, seed pulses are obtained for the $\mathrm{KrF}$ amplifier module which is the key component of the system. This module is a modified version of a Lambda Physik NovaLine laser. In a three-pass amplification scheme, the pulses are amplified up to an energy of $30 \mathrm{~mJ}$ at repetition rates exceeding $350 \mathrm{~Hz}$, resulting in an average power of $10 \mathrm{~W}$ at $248 \mathrm{~nm}$ [8]. The results on UV-femtosecond laser processing shown in Section 3 have been obtained using a predecessor system with 500 femtoseconds pulse duration.

\subsection{Beam Delivery}

Excimer laser processing is usually carried out in a mask projection configuration. A mask consisting of transparent and opaque (or reflective) areas is illuminated by the flat top laser beam. Using a lens or a complex imaging system, the mask is projected on the surface of the work piece, so that the pattern of the mask is reproduced (usually demagnified) in this plane leading to material ablation in the irradiated regions at sufficiently high fluence.

Mask projection is known to be a well-suited irradiation strategy for simultaneous treatment of extended sample areas. On the other hand, multiple beam interference has shown to be very effective in creating a great variety of periodic nanostructures [9-11]. But also a combination of these two techniques allows the fabrication of well-defined, versatile periodic nanostructures over large sample areas $[12,13]$. To create a linear grating pattern, for example, a transmission grating is placed in the mask position, and this mask plane is imaged onto the sample surface using a lens or a microscope objective. By using a suitable arrangement of apertures, distinct diffraction orders can be transmitted while others are blocked. This way the simplest case of two beam interference can be realized, even with laser beams of limited spatial and temporal coherence.

\subsection{Irradiation Configurations}

In principle, there are two different methods to induce ablative removal of a film from a substrate. Either the beam is directed head-on towards the film leading to "front-side 


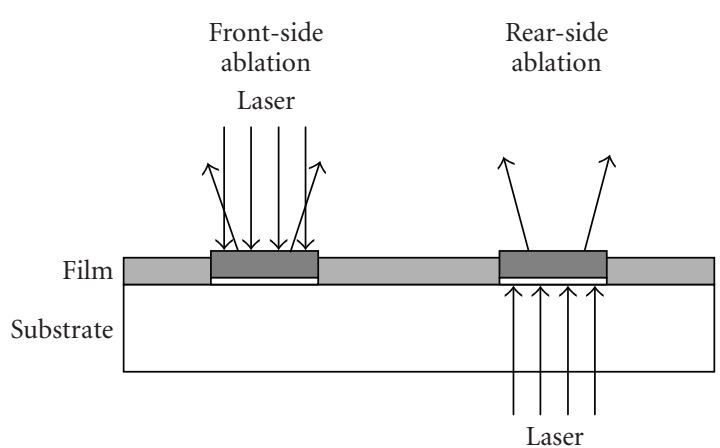

Figure 2: Front-side and rear-side laser ablation of coatings.

ablation," or the beam hits the film from the other side after passing through the substrate "rear-side ablation" (Figure 2). Rear-side ablation is possible, if the substrate is sufficiently transparent at the laser wavelength. For standard excimer laser wavelengths, this is the case, for example, for UV-grade fused silica.

In the case of front-side ablation, depending on laser fluence, film thickness, and absorption properties of film and substrate, the whole film or part of it can be ablated. If the film consists of a layer stack, it is even possible to ablate layer after layer by successive laser pulses [14]. In the case of rear-side irradiation with sufficient fluence, the whole film or layer stack is ablated with a single-laser pulse. The advantage of this method is that due to the forward transfer of the material in the direction of the laser beam, there is no possibility for the interaction of the laser radiation with already ablated fragments. In the case of front-side ablation, this interaction can lead to considerable debris formation around the ablated area, if no countermeasures are applied. Furthermore the required laser fluence for complete ablation is much higher and the edge quality is lower for front-side ablation.

\subsection{Materials and Material Transformation}

The materials of the coatings studied are mainly transparent metal oxides like $\mathrm{Al}_{2} \mathrm{O}_{3}, \mathrm{HfO}_{2}, \mathrm{Ta}_{2} \mathrm{O}_{5}, \mathrm{Nb}_{2} \mathrm{O}_{5}$ with absorption edges somewhere in the UV. As a sufficiently high absorption is a prerequisite for precise ablation, special care has to be taken when selecting the laser wavelength. The applied laser wavelength should be below the absorption edge of the material. $\mathrm{Ta}_{2} \mathrm{O}_{5}, \mathrm{Nb}_{2} \mathrm{O}_{5}$ are transparent above about $300 \mathrm{~nm}$, therefore, for ablation, a shorter wavelength, for example, $248 \mathrm{~nm}$ is required. $\mathrm{HfO}_{2}$ is transparent down to about $220 \mathrm{~nm}$, so that $193 \mathrm{~nm}$ should be used for ablation. $\mathrm{Al}_{2} \mathrm{O}_{3}$ and $\mathrm{SiO}_{2}$ are transparent even at $193 \mathrm{~nm}$, so the use of a $157 \mathrm{~nm}$ radiation is necessary. An alternative approach is the following: instead of an $\mathrm{SiO}_{2}$-coating, a silicon monoxide $(\mathrm{SiO})$ or at least a substoichiometric silicon oxide $\left(\mathrm{SiO}_{x}\right.$, $x<2$ ) is deposited. These materials are UV-absorbing and can therefore be easily processed using $193 \mathrm{~nm}$ or $248 \mathrm{~nm}$ excimer lasers. After patterning, the remaining $\mathrm{SiO}_{x}$ is oxidised to $\mathrm{SiO}_{2}$ by heating it in air to obtain the desired functionality of the patterned coating (Figure 3 ). The ability
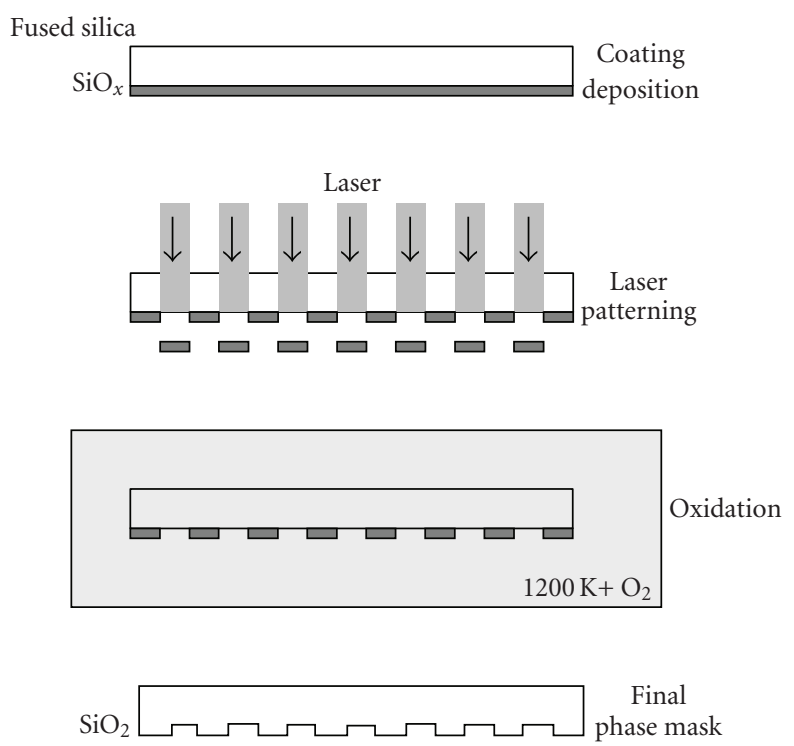

FIgUre 3: Processing scheme for patterning of SiO-coating with subsequent oxidation to $\mathrm{SiO}_{2}$.

of UV laser radiation at $248 \mathrm{~nm}$ to cause ablation of $\mathrm{SiO}_{x}$ films has already been demonstrated long time ago [15].

\section{Results and Discussion}

\subsection{Single Layer Coatings}

Figure 4 shows the results of rear-side ablation of a single silicon monoxide layer. Clean ablation with sharp edges and nearly without redeposition of debris is observed. The ablation depth as a function of the laser fluence for a $285 \mathrm{~nm}$ thick SiO-layer on fused silica is displayed in Figure 5. Within a fluence range of about 200 to $600 \mathrm{~mJ} / \mathrm{cm}^{2}$ the complete layer is ablated with a single-laser pulse (the ablation depth is constant and corresponds to the film thickness). Above this process window, at about $1 \mathrm{~J} / \mathrm{cm}^{2}$ the fused silica substrate is ablated, too. These observations can be explained by treating the layer-substrate boundary as predetermined end points, so that precise depth control and a very smooth surface can be achieved, even if the laser beam is somewhat inhomogeneous within the limits given by the process window. This behavior is similar to that observed in early work on ablating thin metal films with an excimer laser [16], which showed good potential resolution with liftoff through heat flow to the interface.

\subsection{Diffractive Phase Elements}

As an example for the patterning of a transparent film on a transparent substrate, the fabrication of a binary diffractive phase element (DPE) is demonstrated. DPEs are very attractive for beam shaping because of their basically lossless operation. They can be implemented by a (pixelated) surface profile on a transparent optical material and can be applied for beam homogenization, beam splitting or efficient 


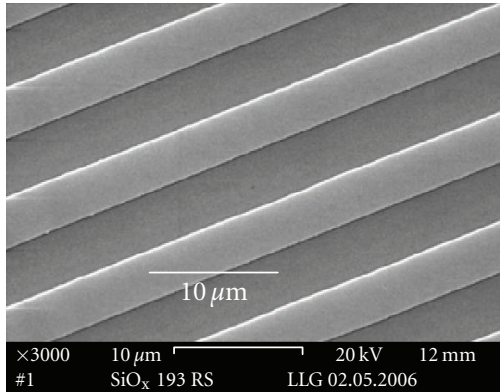

(a)

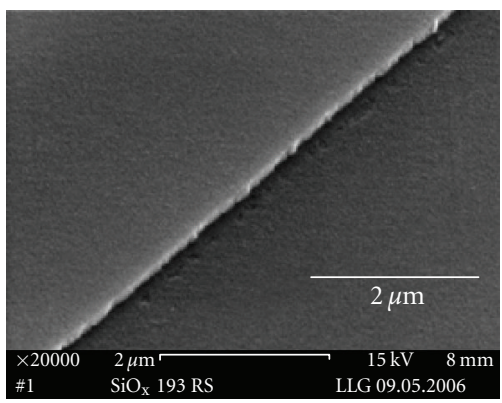

(b)

Figure 4: $175 \mathrm{~nm} \mathrm{SiO}$ on fused silica, rear-side ablation, $193 \mathrm{~nm}$, 20 nanoseconds, $540 \mathrm{~mJ} / \mathrm{cm}^{2}, 1$ pulse.

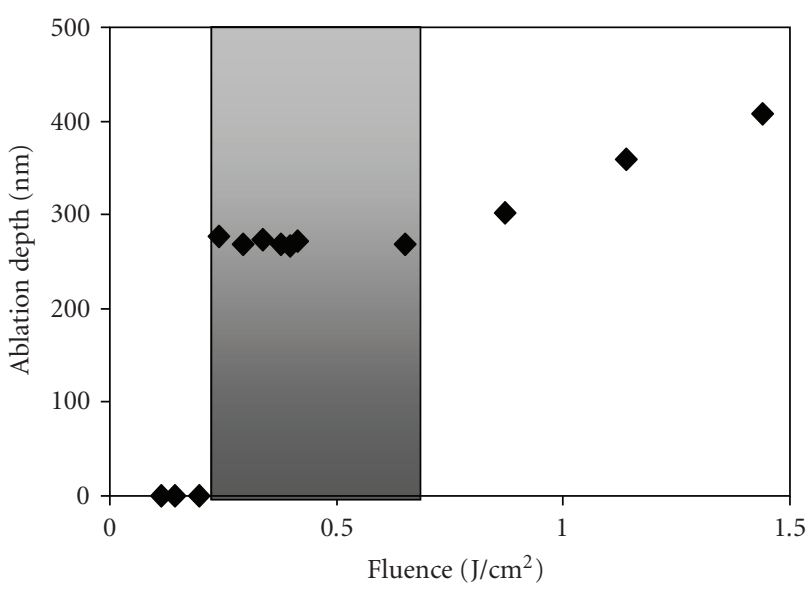

FIGURE 5: Ablation depth versus fluence of $193 \mathrm{~nm}$-single pulse rear side ablation of a $285 \mathrm{~nm}$ thick $\mathrm{SiO}$ layer on fused silica. In the range from 200 to $600 \mathrm{~mJ} / \mathrm{cm}^{2}$ (marked grey), the complete layer is ablated; at fluences above $600 \mathrm{~mJ} / \mathrm{cm}^{2}$, in addition material from the substrate is ablated.

mask illumination. A binary phase element for operation at a UV-wavelength $\lambda$ can be made by ablation patterning a UV-transparent oxide film with a refractive index $n$ and a thickness of $D=\lambda /[2(n-1)]$. Pixel sizes of about $10 \times$ $10 \mu \mathrm{m}^{2}$ are convenient for fabrication and application. The ablation process can be performed pixel by pixel according to thecalculated DPE design or by creating the whole structure at the same time using a mask. For applications of the DPE in the near $\mathrm{UV}, \mathrm{Ta}_{2} \mathrm{O}_{5}$ is an adequate material. $\mathrm{Ta}_{2} \mathrm{O}_{5}$ absorbs

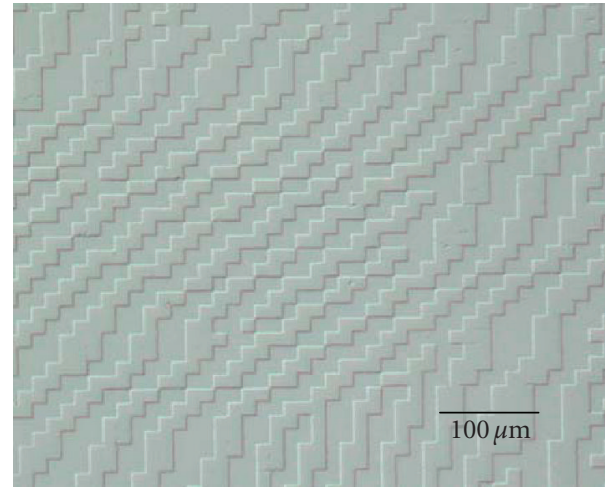

FIGURE 6: $\mathrm{SiO}_{2}$-diffractive phase element made by single-pulse rearside ablation of $\mathrm{SiO}$ at $248 \mathrm{~nm}, 25$ nanoseconds, $350 \mathrm{~mJ} / \mathrm{cm}^{2}$, and subsequent oxidation.

at $248 \mathrm{~nm}$ sufficiently, to be patterned by ablation, but is transparent at $308 \mathrm{~nm}$. This means that a DPE for use at $308 \mathrm{~nm}$ can be fabricated using $248 \mathrm{~nm}$ [17].

For fabricating a phase element to operate in the deep UV, for example, at $193 \mathrm{~nm}$, a film material that is transparent at this wavelength is necessary, for example, $\mathrm{SiO}_{2}$. In this case, the above mentioned two-step process is applied [18]. First, an absorbing $\mathrm{SiO}$ layer is patterned by laser ablation, then the patterned film is oxidised to UV-transparent $\mathrm{SiO}_{2}$. Figure 6 shows a DPE made by this method.

\subsection{Multilayer Coatings}

A typical application for the patterning of multilayers for optical applications is the fabrication of dielectric optical masks. Such multilayer stacks, for example, of alternating $\mathrm{HfO}_{2}$ - (high refractive index) and $\mathrm{SiO}_{2}$-layers (low refractive index) can be ablated by an ArF-excimer laser, because $\mathrm{HfO}_{2}$ is absorbing at $193 \mathrm{~nm}$. Although the thickness of the film is more than $1 \mu \mathrm{m}$, under certain conditions sub- $\mu \mathrm{m}$ edge definition is achieved in the case of rear side ablation.

If both materials of the dielectric layer stack are transparent at $193 \mathrm{~nm}$, the ablation of these systems has to be performed either at even shorter wavelengths (Vacuum-UV) [19], or with an absorbing subsidiary layer. Thus dielectric mirrors with high reflectivity at $193 \mathrm{~nm}$ consisting of a stack of alternating $\mathrm{SiO}_{2}$ - and $\mathrm{Al}_{2} \mathrm{O}_{3}$-layers were patterned by depositing a $193 \mathrm{~nm}$ absorbing $\mathrm{HfO}_{2}$ - or SiO-layer between substrate and HR-stack and ablating in a rear side configuration (Figure 7) [20].

Dielectric masks fabricated by this method can be applied for high intensity laser applications, where metal masks ( $\mathrm{Cr}$ on quartz) would be easily damaged. It is even possible to fabricate grey-level masks by ablating only a defined number of single layers instead of the whole stack [14]. As this process works only by front-side ablation, the edge definition of the ablated structures is limited. To achieve high-precision edges even in the case of front-side ablation, the UV-femtosecond laser system was applied. Figure 8 shows such an ablation 


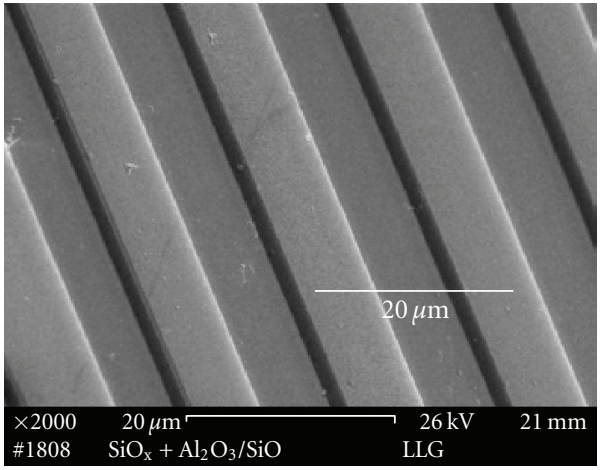

Figure 7: $\mathrm{Al}_{2} \mathrm{O}_{3} / \mathrm{SiO}_{2}$-multilayer stack with underlying $\mathrm{SiO}$ absorber layer on fused silica, rear side ablation, $193 \mathrm{~nm}$, 20 nanoseconds, $500 \mathrm{~mJ} / \mathrm{cm}^{2}, 1$ pulse.

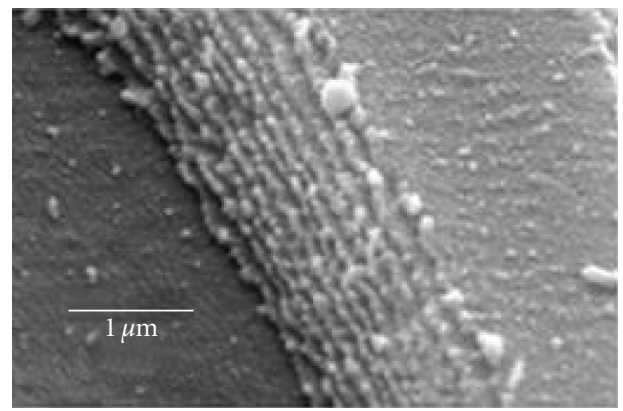

Figure 8: $\mathrm{HfO}_{2} / \mathrm{SiO}_{2}$-multilayer stack on fused silica, front-side ablation, $248 \mathrm{~nm}, 500$ femtoseconds, multipulse irradiation.

edge, where the multilayer structure of the stack can be clearly resolved.

\subsection{High-Resolution Patterning}

Patterning a thin film with high $(\mathrm{sub}-\mu \mathrm{m})$ resolution is desirable, for instance, for the fabrication of optical gratings. To achieve the required optical resolution at high-fluence levels, a mask projection setup using a reflective objective is suitable. The laser pulse duration has to be limited, so that thermal diffusion of the energy coupled into the film does not lead to blurring of the submicron pattern. For metal films, pulse durations as short as 50 ps already lead to diminished structure resolution [21]. For polymer films with their low-thermal diffusivity, comparatively long nanosecond pulses can be applied, but metal oxide films exhibit rather high-thermal diffusivities, so that short pulses are required. Then it is possible to adjust the depth of the pattern in the film by the number of pulses, so that the film can be ablated partly or completely down to the substrate (front side ablation). Figure 9 shows a grating with $500 \mathrm{~nm}$ period made in a $\mathrm{Ta}_{2} \mathrm{O}_{5}$-film with the described ultrashort pulse excimer laser. Such gratings may by applied for coupling light into planar waveguide, or for the so-called grating waveguide structures, which are used, for example, for biosensors based on fluorescence detection [22-24]. Also SiO

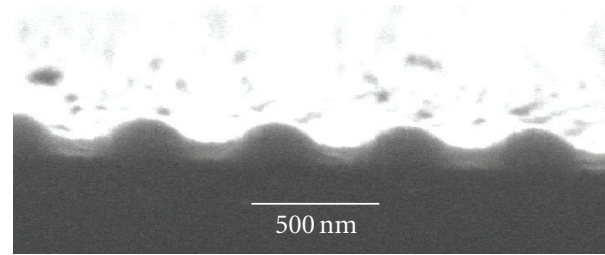

Figure 9: $150 \mathrm{~nm} \mathrm{Ta}_{2} \mathrm{O}_{5}$ on glass, front side ablation, $248 \mathrm{~nm}$, 500 femtoseconds, $300 \mathrm{~mJ} / \mathrm{cm}^{2}, 2$ pulses.

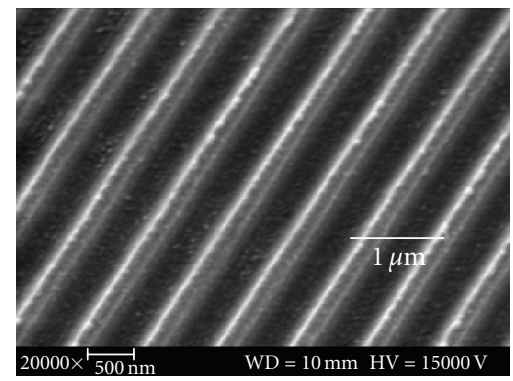

(a)

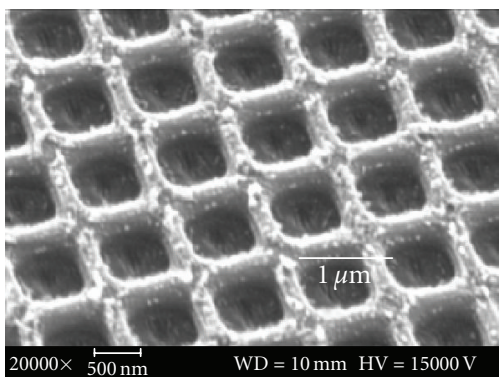

(b)

FIGURE 10: $175 \mathrm{~nm} \mathrm{SiO}$ on fused silica, front side ablation at $248 \mathrm{~nm}$, 500 femtoseconds.

layers can be patterned with high resolution using this UV-fs laser system (Figure 10).

\section{Summary}

UV-transparent inorganic oxide layers are patterned with excimer laser radiation with high precision. Complete layer stacks or only parts of them are ablated depending on fluence and irradiation conditions. To achieve high spatial resolution, a UV-fs-laser system is applied. Nonabsorbing layers are patterned by laser processing of an absorbing substoichiometric "precursor" layer and subsequent oxidation. Thus, dielectric masks, pixelated diffractive phase elements, and submicron gratings are micro fabricated.

\section{Acknowledgment}

The financial support from the BMWi of Germany (grant no. $16 \mathrm{IN} 0395)$ is gratefully acknowledged. 


\section{References}

[1] N. Kaiser and H. K. Pulker, Eds., Optical Interference Coatings, Springer, Berlin, Germany, 2003.

[2] D. Basting and G. Marowsky, Eds., Excimer Laser Technology, Springer, Berlin, Germany, 2005.

[3] D. Bäuerle, Laser Processing and Chemistry, Springer, Berlin, Germany, 3rd edition, 2000.

[4] J. Ihlemann and K. Rubahn, "Excimer laser micro machining: fabrication and applications of dielectric masks," Applied Surface Science, vol. 154-155, pp. 587-592, 2000.

[5] G. P. Behrmann and M. T. Duignan, "Excimer laser micromachining for rapid fabrication of diffractive optical elements," Applied Optics, vol. 36, no. 20, pp. 4666-4674, 1997.

[6] E. Matthias, M. Reichling, J. Siegel, et al., "The influence of thermal diffusion on laser ablation of metal films," Applied Physics A, vol. 58, no. 2, pp. 129-136, 1994.

[7] J. Ihlemann and B. Wolff-Rottke, "Excimer laser ablation patterning of dielectric layers," Applied Surface Science, vol. 86, no. 1-4, pp. 228-233, 1995.

[8] J. Békési, S. Szatmári, P. Simon, and G. Marowsky, “Table-top $\mathrm{KrF}$ amplifier delivering $270 \mathrm{fs}$ output pulses with over $9 \mathrm{~W}$ average power at $300 \mathrm{~Hz}$," Applied Physics B, vol. 75, no. 4-5, pp. 521-524, 2002.

[9] H. M. Phillips and R. A. Sauerbrey, "Excimer-laser-produced nanostructures in polymers," Optical Engineering, vol. 32, no. 10, pp. 2424-2436, 1993.

[10] S. Pissadakis, L. Reekie, M. Hempstead, M. N. Zervas, and J. S. Wilkinson, "Ablated gratings on borosilicate glass by $193-\mathrm{nm}$ excimer laser radiation," Applied Physics A, vol. 69, no. 7, pp. S739-S741, 1999.

[11] T. Kondo, S. Matsuo, S. Juodkazis, V. Mizeikis, and H. Misawa, "Multiphoton fabrication of periodic structures by multibeam interference of femtosecond pulses," Applied Physics Letters, vol. 82, no. 17, pp. 2758-2760, 2003.

[12] J.-H. Klein-Wiele and P. Simon, "Fabrication of periodic nanostructures by phase-controlled multiple-beam interference," Applied Physics Letters, vol. 83, no. 23, pp. 4707-4709, 2003.

[13] J.-H. Klein-Wiele, J. Békési, J. Ihlemann, and P. Simon, "High speed fabrication of periodic nanostructures," in Proceedings of the 3rd International WLT-Conference Lasers in Manufacturing (LIM '05), p. 477, Munich, Germany, June 2005.

[14] K. Rubahn and J. Ihlemann, "Graded transmission dielectric optical masks by laser ablation," Applied Surface Science, vol. 127-129, pp. 881-884, 1998.

[15] C. Fiori and R. A. B. Devine, "High resolution ultraviolet photoablation of $\mathrm{SiO}_{x}$ films," Applied Physics Letters, vol. 47, no. 4, pp. 361-362, 1985.

[16] J. E. Andrew, P. E. Dyer, R. D. Greenough, and P. H. Key, "Metal film removal and patterning using a XeCl laser," Applied Physics Letters, vol. 43, no. 11, pp. 1076-1078, 1983.

[17] J. Ihlemann and D. Schäfer, "Fabrication of diffractive phase elements for the UV-range by laser ablation patterning of dielectric layers," Applied Surface Science, vol. 197-198, pp. 856-861, 2002.

[18] M. Schulz-Ruhtenberg, J. Ihlemann, and J. Heber, "Laser patterning of $\mathrm{SiO}_{x}$-layers for the fabrication of UV diffractive phase elements," Applied Surface Science, vol. 248, no. 1-4, pp. 190-195, 2005.

[19] D. Schäfer, J. Ihlemann, G. Marowsky, and P. R. Herman, "F2laser ablation patterning of dielectric layers," Applied Physics A, vol. 72, no. 3, pp. 377-379, 2001.
[20] J. Ihlemann, K. Rubahn, and R. Thielsch, "Laser ablation patterning of dielectric layer stacks for 193-nm mask fabrication," in Second International Symposium on Laser Precision Microfabrication, vol. 4426 of Proceedings of SPIE, pp. 437-440, Singapore, May 2002.

[21] P. Simon and J. Ihlemann, "Machining of submicron structures on metals and semiconductors by ultrashort UV-laser pulses," Applied Physics A, vol. 63, no. 5, pp. 505-508, 1996.

[22] F. Beinhorn, J. Ihlemann, P. Simon, et al., "Sub-micron grating formation in $\mathrm{Ta}_{2} \mathrm{O}_{5}$-waveguides by femtosecond UV-laser ablation," Applied Surface Science, vol. 138-139, no. 1-4, pp. 107-110, 1999.

[23] S. Pissadakis, M. N. Zervas, L. Reekie, and J. S. Wilkinson, "High-reflectivity Bragg gratings fabricated by 248-nm excimer laser holographic ablation in thin $\mathrm{Ta}_{2} \mathrm{O}_{5}$ films overlaid on glass waveguides," Applied Physics A, vol. 79, no. 4-6, pp. 1093-1096, 2004.

[24] C. Kappel, A. Selle, T. Fricke-Begemann, M. A. Bader, and G. Marowsky, "Giant enhancement of two-photon fluorescence induced by resonant double grating waveguide structures," Applied Physics B, vol. 79, no. 5, pp. 531-534, 2004. 